\title{
Note
}

\section{Development of backcross recombinant inbred lines between Oryza sativa Nipponbare and $O$. rufipogon and QTL detection on drought tolerance}

\author{
Pham Thien Thanh, Phuong Dang Thai Phan, Naoki Mori, Ryo Ishikawa and Takashige Ishii* \\ Graduate School of Agricultural Science, Kobe University, 1-1 Rokkodai, Nada, Kobe, Hyogo 657-8501, Japan
}

\begin{abstract}
Backcross populations at advanced generations are useful in evaluating the agronomic traits from unadapted germplasms, such as wild species. In this study, we generated 159 backcross recombinant inbred lines (BRILs) at the $\mathrm{BC}_{2} \mathrm{~F}_{8}$ generation between Oryza sativa Nipponbare (a recurrent parent) and O. rufipogon W630 (a donor parent). These BRILs were found to have nearly homozygous genome constitutions based on the marker genotypes at 180 SSR loci covering whole chromosomes. Since their selfed progenies have almost identical genotypes in each line, they were further used for QTL analysis of drought tolerance. Out of six QTLs detected, three were found to have wild alleles with favorable effects on drought tolerance. The present BRILs with known marker data can be applied to more QTL analyses of traits specific to wild species.
\end{abstract}

Key Words: rice, Oryza rufipogon, backcross recombinant inbred lines, QTL analysis, drought tolerance.

\section{Introduction}

The genus Oryza comprises two cultivated species and more than 20 wild species (Vaughan 1994). Based on the studies on morphological traits and hybrid fertility, Asian common rice (Oryza sativa) was found to have originated from the Asian wild species, O. rufipogon (Morishima et al. 1963, Oka and Chang 1961). Since O. rufipogon has a higher cross-affinity with $O$. sativa than other wild species, it provides most accessible wild genetic resources for improvement of rice cultivars. In fact, many useful traits have been identified and transferred to elite breeding lines (Brar and Khush 1997). These traits are usually controlled by single genes and can be easily identified by phenotypic screening. On the other hand, quantitative traits, such as plant size and yield, are associated with many genes, and their manner of inheritance seems to be very complicated. Tanksley and Nelson (1996) proposed an advanced backcross QTL analysis as a molecular breeding method to identify and transfer agronomically useful QTLs from unadapted germplasms. Using $\mathrm{BC}_{2} \mathrm{~F}_{1}$ and $\mathrm{BC}_{2} \mathrm{~F}_{2}$ populations, many wild QTL alleles have been found to have the potential of substantially increasing the quality or productivity of cultivated tomato and rice species (Tanksley et al. 1996, Xiao et al. 1998). However, the marker data used for QTL analysis were applicable only with the population examined. Since heterozygous chromosomal segments are still maintained in the backcross

Communicated by Duncan Vaughan

Received Septembner 13, 2010. Accepted November 10, 2010.

*Corresponding author (e-mail: tishii@kobe-u.ac.jp) plants at $\mathrm{BC}_{2} \mathrm{~F}_{1}$ and $\mathrm{BC}_{2} \mathrm{~F}_{2}$ generations, their selfed progenies show further segregation of genotypes.

Recombinant inbred lines (RILs) are produced by continually selfing the progeny of each $\mathrm{F}_{2}$ plant. They constitute a permanent population in which segregation is fixed. In rice, several RILs were produced between $O$. sativa and $O$. rufipogon, and QTL analyses of domestication-related traits were successfully performed (Cai and Morishima 2002, Onishi et al. 2007). However, it is difficult to evaluate agronomical traits with RILs because large morphological variations are expected to occur among RILs and many undesirable wild traits would mask the effects of beneficial QTL alleles.

In this study, we generated backcross recombinant inbred lines (BRILs) at the $\mathrm{BC}_{2} \mathrm{~F}_{8}$ generation between $O$. sativa Nipponbare (a recurrent parent) and O. rufipogon W630 (a donor parent). These BRILs were found to have nearly homozygous genomes based on the fixation levels of marker genotypes at SSR loci. Since their selfed progenies have almost identical genotypes in each line, they can be applied for the trait evaluation that requires many individuals or replications. O. rufipogon is a wild species naturally surviving in tropical Asian countries, and it sometimes encounters severe natural stresses such as drought. In a preliminary experiment, we found $O$. rufipogon W630 had high degree of drought tolerance. Therefore, the BRILs having small wild chromosomal segments in the genetic background of O. sativa Nipponbare were further used for QTL analysis of drought tolerance to survey the useful wild alleles. 


\section{Materials and Methods}

\section{Plant materials}

A Japonica rice cultivar, O. sativa Nipponbare, and a wild annual accession of $O$. rufipogon W630 from Myanmar having high degrees of drought tolerance were used in this study. The wild seeds were provided by the National Institute of Genetics, Japan. BRILs were produced as follows: A single wild plant of $O$. rufipogon $\mathrm{W} 630$ was crossed twice with $O$. sativa Nipponbare, and $18 \mathrm{BC}_{1} \mathrm{~F}_{1}$ plants were obtained. They were further backcrossed, and $216 \mathrm{BC}_{2} \mathrm{~F}_{1}$ plants (12 plants each from $18 \mathrm{BC}_{1} \mathrm{~F}_{1}$ plants) were generated. From them, BRILs at the $\mathrm{BC}_{2} \mathrm{~F}_{8}$ generation were developed by the single-seed-descendant method. The plants were not artificially selected during the selfing process, but almost 50 lines were eliminated because of weak growth and sterility. As a result, 159 BRILs, which theoretically contain $1 / 8$ of the wild genome in the genetic background of $O$. sativa Nipponbare, were generated.

\section{Determination of marker genotypes at SSR loci}

DNA was extracted from 159 BRILs using the potassium acetate method as described by Dellaporta et al. (1983). In total, 180 polymorphic SSR markers between $O$. sativa Nipponbare and O. rufipogon W630 were selected to survey the BRIL genotypes in 12 chromosomes. The total map size of these markers was $1362 \mathrm{cM}$, with an average marker interval of $8.2 \mathrm{cM}$. The primer sequences of these SSR markers were obtained from the Gramene database (http:// www.gramene.org/) by Liang et al. (2008). PCR was carried out in a $25-\mu 1$ reaction, and the amplified products were electrophoresed in $4 \%$ polyacrylamide denaturing gel with $0.5 \times$ TBE buffer. The microsatellite banding patterns were visualized using the silver staining method as described by Panaud et al. (1996).

\section{Evaluation of drought tolerance}

Drought tolerance evaluation was carried out in 159 BRILs (or $\mathrm{BC}_{2} \mathrm{~F}_{9}$ plants) and their parents. The experiments were performed in the university greenhouse to avoid rainwater. An upland cultivar of IRAT13 was also included as a drought-resistant control. The germinated seeds were sown in the seedling cases, and three-week-old seedlings were transplanted at intervals of $8 \mathrm{~cm}$ in a soil plot of $0.8 \mathrm{~m} \times 5.5 \mathrm{~m}$ with three replications. The plot was surrounded by a cement frame, and equal moisture levels in the plot were checked by a model DM- 8 tensiometer (Takemura Co., Japan). After supplying the water for 30 days, irrigation was withheld. On the 12th day of drought treatment, some plants started to show rolling leaves. On the 21 st day, when the leaf-rolling phenotypes were highly varied among the BRILs, the degree of leaf rolling was visually scored at midday on a scale of 0-9 (0: unrolled, 9: tightly rolled) according to the scores of the Standard Evaluation System for rice (INGER 1996). The BRILs were continuously maintained under drought stress for the next 18 days to induce leaf dry- ing. Irrigation was restarted, and after 10 days the drying of leaves was scored on a scale of 0-9 (0: no symptoms, 9: all leaves apparently dead) (INGER 1996). For both traits, average scores of three replications were calculated for all lines.

\section{QTL analysis}

Based on the marker and trait data, QTL analysis for drought tolerance was carried out. Putative QTLs were estimated by composite interval mapping using WinQTL Cartographer ver. 2.5 software (Wang et al. 2007). The optimal $\log$ of odds (LOD) threshold values obtained from WinQTL Cartographer (with the permutation value set at 1,000) were used to determine the presence of a putative QTL. The percentages of variation explained by the QTL and the additive effect were also estimated using the software.

\section{Results and Discussion}

\section{Genome composition of $\mathrm{BC}_{2}$ backcross inbred lines}

BRILs at the $\mathrm{BC}_{2} \mathrm{~F}_{8}$ generation were expected to have the genetic constitution of the recurrent cultivar, $O$. sativa Nipponbare, with the exception of small substitutions of chromosome segments from the wild accession, O. rufipogon W630. Theoretically, these BRILs may have $1 / 8$ (12.5\%) of the wild genome in the genetic background of Nipponbare. Based on the marker genotypes at 180 microsatellite loci on 12 rice chromosomes covering $1,362 \mathrm{cM}$, the wild chromosomal segments in each line were estimated. The frequency distribution of the proportion of wild genome introgressed into BRILs is shown in Fig. 1. The percentages of wild genome ranged from $0.0 \%$ to $23.6 \%$, with a mean of $11.3 \%$. This value is close to the theoretical ratio of $12.5 \%$. Compared with lines having specific wild chromosomal segments, such as chromosome segment substitution lines and introgression lines, the BRILs have various combinations of wild chromosomal blocks in the genome. Therefore, they are good materials to investigate interactions between wild genes/loci.

The numbers of the lines having wild homozygous alleles at 180 microsatellite loci were examined. The minimum and maximum numbers of the lines having wild homozygous alleles were three and 39, respectively. This shows that no loci were fixed with only Nipponbare alleles, and a set of BRILs used here may carry the complete genomic regions of W630. By the single-seed-descendant method, 159 BRILs were developed from the $\mathrm{BC}_{2} \mathrm{~F}_{1}$ plants that originated from 18 $\mathrm{BC}_{1} \mathrm{~F}_{1}$ plants. Although we can not directly examine the segregation distortion among BRILs because of the bottleneck effect at the $\mathrm{BC}_{1} \mathrm{~F}_{1}$ generation, the following three wild chromosomal regions were found to be covered by a few BRILs (3-5 lines): RM259-RM35 region on chromosome 1, RM417-RM142 on chromosome 4, and RM219-RM321 on chromosome 9 . These suggest that some wild genes associated with semi-sterility or hybrid weakness are located in the regions. In this study, the genotypes at 28,620 loci (180 loci $\times 159$ lines) were surveyed, but 21 could not be 


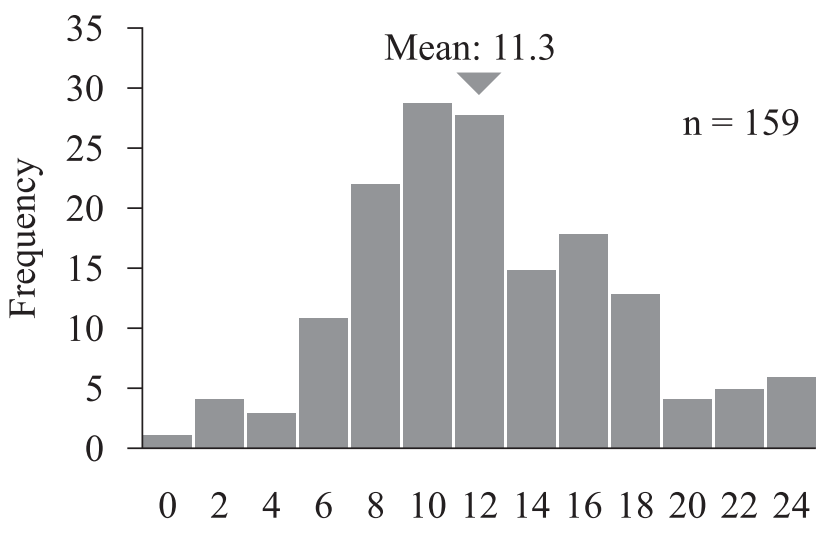

Percentage of $O$. rufipogon genome (\%)

Fig. 1. Frequency distribution of percentage of $O$. rufipogon genome found in the backcross recombinant inbred lines between $O$. sativa Nipponbare and O. rufipogon W630.

determined. The remaining 28,599 loci consist of 25,277 Nipponbare homozygous, 161 heterozygous and 3,161 wild W630 homozygous loci. The homozygous fixation value was $99.4 \% ;(25,277+3,161) / 28,599$. This result confirmed that the BRILs at the $\mathrm{BC}_{2} \mathrm{~F}_{8}$ generation are almost pure lines and their progenies can be repeatedly used for the trait evaluation that requires many individuals or replications.

\section{QTL analysis for drought tolerance}

Drought tolerance evaluation was carried out using 159 BRILs and their parents in the university greenhouse with three replications. O. rufipogon $\mathrm{W} 630$ was more tolerant to drought (1.7 and 1.0 for average leaf rolling and leaf drying scores, respectively) than $O$. sativa Nipponbare (9.0 and 6.3 for average leaf rolling and leaf drying scores, respectively). The degree of tolerance of W630 was slightly stronger than that of the drought resistant control cultivar (IRAT13) in this experiment. The frequency distributions for leaf rolling and leaf drying in BRILs are shown in Fig. 2A and 2B, respectively. Using these trait data, QTL analysis for drought tolerance was carried out. In total, four and two QTLs were found for leaf rolling and leaf drying, respectively (Table 1 and Fig. 3). Their LOD score varied from 3.56 to 7.92 , and individual QTL explained variance ranged from $6.8 \%$ to $23.0 \%$. Desirable wild alleles decreasing drought scores were detected at one locus for leaf rolling in the RM243-RM129 region on chromosome 1 and two for leaf drying in the RM580-RM9 and RM249-RM440 regions on chromosomes 1 and 5, respectively. These useful wild QTL regions on chromosome 1 for both traits were partly overlapping and were close to the QTL for leaf drying reported by Price et al. (2002). In the similar region of RM249-RM440 on chromosome 5, Yue et al. (2005) detected a leaf rolling QTL with a drought-tolerant allele from IRAT109 (an upland rice cultivar). Although they found QTLs near the regions where we detected, their plant materials and drought treatment procedures were different from those used in this study. More
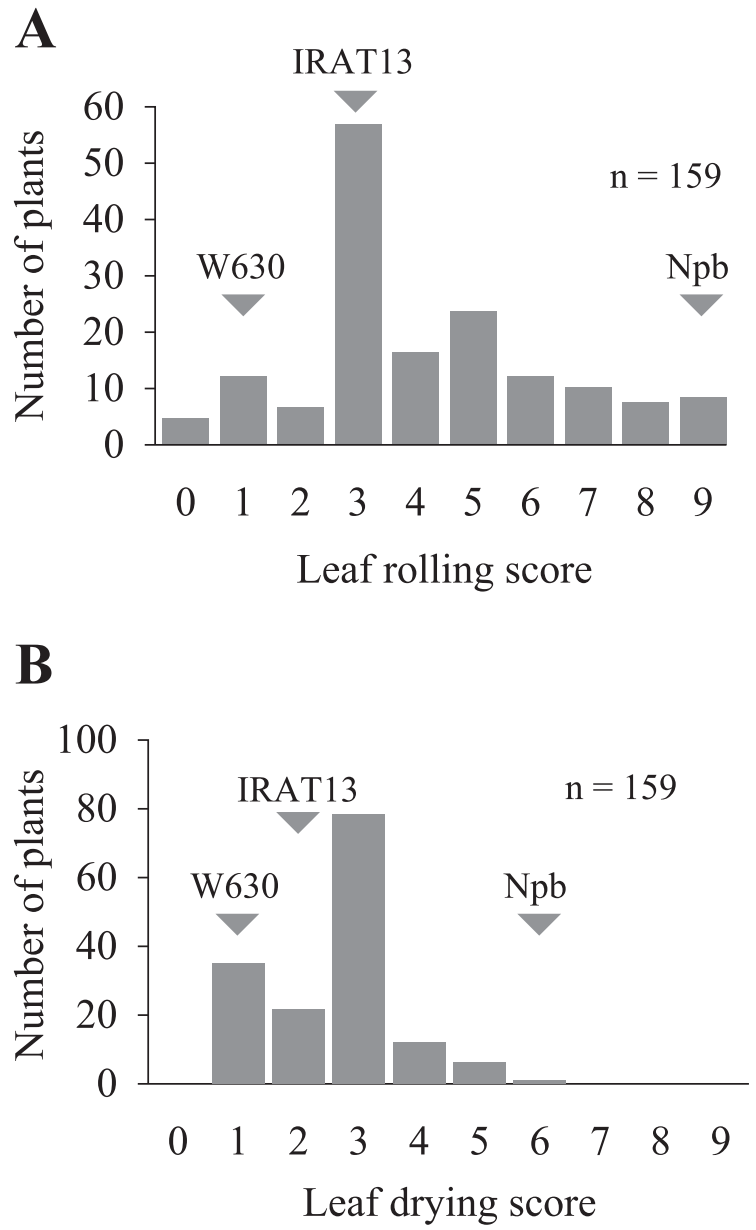

Fig. 2. Frequency distribution of drought tolerance in the backcross recombinant inbred lines between $O$. sativa Nipponbare and O. rufipogon W630. (A) Leaf rolling. (B) Leaf drying. Npb: O. sativa Nipponbare. W630: O. rufipogon W630.
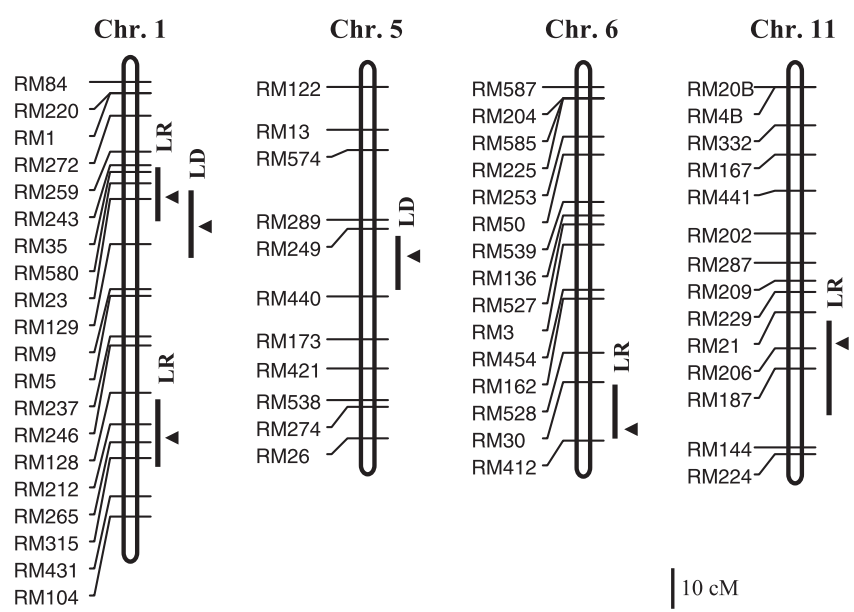

Fig. 3. Chromosome positions of putative QTLs controlling drought tolerance (LR: leaf rolling, LD: leaf drying) detected in the backcross recombinant inbred lines between $O$. sativa Nipponbare and O. rufipogon W630. QTL positions are represented as bars with the LOD peaks shown by arrows. 
Table 1. Putative QTLs for drought tolerance (LR: leaf rolling, LD: leaf drying) detected in the backcross recombinant inbred lines between $O$. sativa Nipponbare and $O$. rufipogon W630

\begin{tabular}{lrlllrrr}
\hline \hline Trait & Chr. & QTL region & $\begin{array}{l}\text { Nearest } \\
\text { marker }\end{array}$ & LOD & $\begin{array}{r}\mathrm{PV}^{a} \\
(\%)\end{array}$ & $\mathrm{A}^{b}$ & Source $^{c}$ \\
\hline LR & 1 & RM243-RM129 & RM23 & 4.51 & 8.9 & -1.12 & $\mathrm{~W}$ \\
& 1 & RM128-RM431 & RM265 & 3.64 & 6.8 & 0.80 & $\mathrm{~N}$ \\
& 6 & RM30-RM412 & RM412 & 7.92 & 23.0 & 1.46 & $\mathrm{~N}$ \\
& 11 & RM21-RM144 & RM206 & 5.62 & 12.9 & 1.11 & $\mathrm{~N}$ \\
\hline LD & 1 & RM580-RM9 & RM129 & 3.93 & 13.1 & -0.59 & $\mathrm{~W}$ \\
& 5 & RM249-RM440 & RM440 & 3.56 & 17.0 & -0.62 & $\mathrm{~W}$
\end{tabular}

${ }^{a}$ Percent phenotypic variance explained by the QTL.

${ }^{b}$ Additive effect of the allele from O. rufipogon W630.

${ }^{c}$ Favorable allele source for drought tolerance (decreasing effects on LR and LD). W: O. rufipogon W630. N: O. sativa Nipponbare.

detailed examination is required at different stages to confirm the useful wild alleles for drought tolerance.

\section{Conclusions}

Advanced backcross populations are useful in evaluating the agronomic traits from unadapted germplasms, such as wild species. In this study, we generated 159 BRILs at the $\mathrm{BC}_{2} \mathrm{~F}_{8}$ generation between $O$. sativa Nipponbare and $O$. rufipogon W630. Their chromosomal information was determined using 180 SSR markers covering whole chromosomes. BRILs were found to have nearly homozygous genome constitutions, and the same genotypes of the lines are easily produced from their selfed progenies without segregation. They can be applied for the trait evaluation that requires many individuals or replications. Besides the QTL analysis on drought tolerance in this study, we are able to carry out more QTL analyses for traits specific to wild species using the same set of BRILs with known marker genotype data.

\section{Acknowledgements}

We thank the Vietnamese Government for funding Ph.D. scholarship to P.T.T. The seeds of O. rufipogon W630 were kindly provided from National Institute of Genetics (Japan) supported by the National BioResource Project (MEXT, Japan). This work was supported in part by a Grant-in-Aid (No. 20580005 to T.I.) from Japanese Society for Promotion of Science (JSPS).

\section{Literature Cited}

Brar,D.S. and G.S.Khush (1997) Alien introgression in rice. Plant Mol. Biol. 35: 35-47.

Cai,H.W. and H.Morishima (2002) QTL clusters reflect character associations in wild and cultivated rice. Theor. Appl. Genet. 104: 1217-1228.

Dellaporta, S.L., H.Wood and J.B.Hicks (1983) A plant DNA minipreparation. Plant Mol. Biol. Rep. 1: 19-21.

INGER (1996) Standard evaluation system for rice. International Rice Research Institute, Manila, p.52.

Liang, C., P. Jaiswal, C. Hebbard, S. Avraham, E.S. Buckler, T. Casstevens, B.Hurwitz, S.McCouch, J.Ni, A.Pujar et al. (2008) Gramene: a growing plant comparative genomics resource. Nucleic Acids Res. 36: 947-953.

Morishima,H., K.Hinata and H.I.Oka (1963) Comparison of modes of evolution of cultivated forms from two wild rice species, Oryza breviligulata and O. perennis. Evolution 17: 170-181.

Oka,H.I. and W.T.Chang (1961) Hybrid swarms between wild and cultivated species, Oryza perennis and O. sativa. Evolution 15: 418-430.

Onishi,K., Y.Horiuchi, N.Ishigoh-Oka, K. Takagi, N. Ichikawa, M. Maruoka and Y.Sano (2007) A QTL cluster for plant architecture and its ecological significance in Asian wild rice. Breed. Sci. 57: $7-16$.

Panaud,O., X.Chen and S.R.McCouch (1996) Development of microsatellite markers and characterization of simple sequence length polymorphism (SSLP) in rice (Oryza sativa L.). Mol. Gen. Genet. 252: 597-607.

Price, A.H., J. Townend, M.P. Jones, A. Audebert and B.Courtois (2002) Mapping QTLs associated with drought avoidance in upland rice grown in the Philippines and West Africa. Plant Mol. Biol. 48: 683-695.

Tanksley,S.D. and J.C.Nelson (1996) Advanced backcross QTL analysis: a method for the simultaneous discovery and transfer of valuable QTLs from unadapted germplasm into elite breeding lines. Theor. Appl. Genet. 92: 191-203.

Tanksley, S.D., S. Grandillo, T.M.Fulton, D.Zamir, Y.Eshed, V. Petiard, J.Lopez and T.Beck-Bunn (1996) Advanced backcross QTL analysis in a cross between an elite processing line of tomato and its wild relative L. pimpinellifolium. Theor. Appl. Genet. 92: 213-224.

Vaughan,D.A. (1994) The wild relatives of rice. International Rice Research Institute, Manila, Philippines, p. 137.

Wang,S., C.J.Basten, P.Gaffney and Z.B.Zeng (2007) Windows QTL Cartographer 2.5. Bioinformatics Research Center, North Carolina State University, USA.

Xiao, J., J.Li, S.Grandillo, S.N.Ahn, L. Yuan, S.D. Tanksley and S.R.McCouch (1998) Identification of trait-improving quantitative trait loci alleles from a wild rice relative, Oryza rufipogon. Genetics 150: 899-909.

Yue,B., L.Xiong, W.Xue, Y.Xing, L.Luo and C.Xu (2005) Genetic analysis for drought resistance of rice at reproductive stage in field with different types of soil. Theor. Appl. Genet. 111: 1127-1136. 